\title{
Glucocorticoids induce autophagy in rat bone marrow mesenchymal stem cells
}

\author{
LONG WANG $^{1}$, JING FAN ${ }^{1}$, YAN-SHUI LIN ${ }^{2}$, YUN-SHAN GUO ${ }^{1}$, BO GAO $^{1}$, QI-YUE SHI ${ }^{1}$,

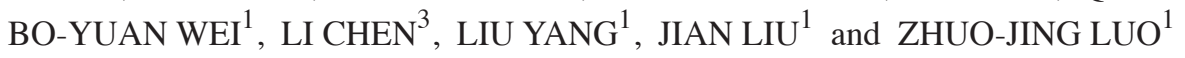 \\ ${ }^{1}$ Department of Orthopedics, Institute of Orthopedic Surgery, Xijing Hospital, Fourth Military Medical University, \\ Xi'an, Shaanxi 710032; ${ }^{2}$ Department of Orthopedics, First Affiliated Hospital, Chengdu Medical College, \\ Chengdu, Sichuan 610500, P.R. China; ${ }^{3}$ Laboratory of Molecular Endocrinology, Odense University Hospital, \\ University of Southern Denmark, 5000 Odense C, Denmark
}

Received October 14, 2013; Accepted October 31, 2014

DOI: $10.3892 / \mathrm{mmr} .2014 .3099$

\begin{abstract}
Glucocorticoid-induced osteoporosis (GIOP) is a widespread clinical complication following glucocorticoid therapy. This irreversible damage to bone-forming and -resorbing cells is essential in the pathogenesis of osteoporosis. Autophagy is a physiological process involved in the regulation of cells and their responses to diverse stimuli, however, the role of autophagy in glucocorticoid-induced damage to bone marrow mesenchymal stem cells (BMSCs) remains unclear. The current study confirmed that glucocorticoid administration impaired the proliferation of BMSCs. Transmission electron microscopy, immunohistochemistry and western blot analysis detected autophagy in vitro and in GIOP model rats (in vivo). With the addition of the autophagy inhibitor 3-methyladenine, the proliferative ability of BMSCs was further reduced, while the number of apoptotic BMSCs was significantly increased. The data suggests that in response to glucocorticoid administration, induced autophagy aids to maintain proliferation and prevent apoptosis of BMSCs. Thus, it is hypothesized that autophagy may be a novel target in the treatment or prevention of osteoporosis.
\end{abstract}

\section{Introduction}

Glucocorticoids have been widely used in the treatment of non-infectious inflammation and autoimmune disorders, however, long-term glucocorticoid therapy has been demonstrated to lead to irreversible bone injury (1). Weinstein (2)

Correspondence to: Professor Zhuo-Jing Luo or Dr Liu Yang, Department of Orthopedics, Institute of Orthopedic Surgery, Xijing Hospital, Fourth Military Medical University, 127 Changle West Road, Xi'an, Shaanxi 710032, P.R. China

E-mail: zjluo@fmmu.edu.cn

E-mail: yangliu@fmmu.edu.cn

Key words: osteoporosis, glucocorticoid, autophagy, bone mesenchymal stem cell, apoptosis reported that the incidence of bone fracture was 30-50\% among patients receiving long-term glucocorticoid therapy; and glucocorticoid-induced osteoporosis (GIOP) is the most common type of secondary osteoporosis (3). Previous studies have suggested that oral glucocorticoids may reduce the proliferation and increase the apoptosis of osteoblasts and osteocytes (4), in addition to prolonging the survival of osteoclasts (5). The balance between bone formation and resorption is interrupted and the risk of bone fracture is increased following glucocorticoid therapy (6).

Bone marrow mesenchymal stem cells (BMSCs) are essential in the maintenance of the dynamic homeostasis of bone tissue, with studies demonstrating that when BMSC proliferation and osteoblastic differentiation are defective, bone mass is reduced $(7,8)$. In addition, Zhou et al (9) indicated that stem cell injury and dysfunction are involved in the pathogenesis of osteoporosis. However, whether human (h)BMSCs from patients with GIOP are damaged and whether glucocorticoid is responsible for these defective hBMSCs remains unclear.

Autophagy is a conserved cellular process that involves the degradation and recycling of dysfunctional and unnecessary cellular components, protein aggregates and intracellular pathogens (10). During the process of autophagy, cytoplasmic targets are enwrapped within autophagosomes and fused with lysosomes, forming autolysosomes. These cytoplasmic constituents are then degraded or recycled (11). This physiological process is essential for the regulation of cells and their responses to diverse stimuli. Several studies have now demonstrated that autophagy is able to regulate the function of osteoclasts (12), osteoblasts (13) and osteocytes (14), suggesting this process to be essential in bone homeostasis.

One study demonstrated that glucocorticoids had a positive effect on osteocyte survival via the induction of autophagy (15). Another study observed that autophagy was able to regulate cell reprogramming. This reduces the level of intercellular reactive oxygen species, thus maintaining the ability of the cell to self-renew during stem cell differentiation (16). However, whether autophagy is involved in glucocorticoid-induced damage to BMSCs remains to be elucidated.

The current study hypothesized that glucocorticoid therapy impairs proliferation and induces autophagy in the BMSCs. In 
addition, glucocorticoid-induced autophagy was hypothesized to be involved in maintaining proliferation and preventing apoptosis in BMSCs.

\section{Materials and methods}

Animals and experimental procedures. A total of 20 female Sprague-Dawley rats (4 months old; average weight, $235 \pm 19.4 \mathrm{~g}$ ) obtained from the Laboratory Animal Centre of Fourth Military Medical University (Xi'an, China) were randomly divided into two groups with five rats in each group. Randomization was performed using a random number table. The groups were divided as follows: i) Placebo, $10 \mathrm{ml} / \mathrm{kg}$ distilled water; and ii) GIOP (prednis), $5 \mathrm{mg} / \mathrm{kg}$ prednisolone (Sigma-Aldrich, St. Louis, MO, USA). All treatments were administered daily via oral gavage, for 8 weeks prior to sacrifice by cervical vertebra luxation. All experimental procedures were approved by the Institutional Ethics Review Board of Xijing Hospital (permission code 20110405-5).

Cell culture. Bone marrow was obtained from the tibia and femur of Sprague-Dawley rats and seeded into $75 \mathrm{~cm}^{2}$ culture flasks with $\alpha$-modified minimum essential media ( $\alpha$-MEM; Thermo Fisher Scientific, Waltham, MA, USA), containing $10 \%$ fetal bovine serum and $1 \%$ penicillin-streptomycin (all from Gibco Life Technologies, Carlsbad, CA, USA) under conditions of $5 \% \mathrm{CO}_{2}$ and $37^{\circ} \mathrm{C}$. When $80 \%$ confluence was reached, the cells were detached using $0.25 \%$ trypsin-EDTA (Gibco Life Technologies) and passaged at the ratio of 1:2. The number of nonadherent hematopoietic cells reduced during consecutive passages. All cells used in the experiment were used at passage 3 .

Cell treatment. The BMSCs were treated with various concentrations $\left(0 \mathrm{M}, 10^{-8} \mathrm{M}, 10^{-7} \mathrm{M}, 10^{-6} \mathrm{M}\right)$ of the glucocorticoid dexamethasone (Dex; Sigma-Aldrich) for $48 \mathrm{~h}$. The autophagy inhibitor, 3-methyladenine (3-MA; Sigma-Aldrich) was added to the culture media with a final concentraion of $5 \mathrm{mM}$ in order to investigate the role and mechanism of autophagy in the proliferation and apoptosis of BMSCs.

Cell proliferation assay. Following Dex treatment, the Cell Counting Kit-8 (CCK-8; Dojindo Molecular Technologies, Inc., Kumamoto, Japan) was used to measure cell growth, in accordance with the manufacturer's instructions, and cells were measured at wavelengths of 450 and $630 \mathrm{~nm}$ using a Thermo Labsystems Multiscan MK-3 enzyme-linked microplate reader (Thermo Fisher Scientific).

Detection of autophagosomes by transmission electron microscopy (TEM). Cells were detached from the plates using $0.25 \%$ trypsin trypsin-EDTA (Gibco Life Technologies) and fixed with $2 \%$ paraformaldehyde/2\% glutaraldehyde (Sigma-Aldrich) in $0.2 \mathrm{M}$ sodium cacodylate buffer (pH 7.4; Sigma-Aldrich). Cell pellets were post-fixed with $1 \%$ (v/v) osmic acid (Sigma-Aldrich) in sodium cacodylate buffer and were stained with $1 \%$ uranyl acetate (Amresco, Solon, OH, USA). Following dehydration, the pellets were embedded in Durcupan (Sigma-Aldrich). Ultrathin sections $(50 \mathrm{~nm})$ were prepared using an Ultrotome Ultracut S (Leica
Microsystems, Wetzlar, Germany) and images were captured with a JEM-1230 transmission electron microscope (JEOL, Ltd., Tokyo, Japan).

Immunofluorescence. Cells were cultured in 4-well chamber slides (Thermo Fisher Scientific) and treated in accordance with the manufacturer's instructions for immunostaining. Cells were fixed in $4 \%$ paraformaldehyde for $15 \mathrm{~min}$, permeabilized with $100 \%$ methanol (Sigma-Aldrich) for 10 min and were then incubated with the primary antibody (1:200; monoclonal rabbit anti-rat LC3B antibody; Cell Signaling Technology, Inc., Danvers, MA, USA) overnight, followed by the secondary antibody (1:200; monoclonal goat anti-rabbit DyLight 594; Abcam, Cambridge, MA, USA) for $1 \mathrm{~h}$. Subsequent to incubation with DAPI $(0.5 \mu \mathrm{g} / \mathrm{ml}$; KeyGEN, Nanjing, China) for $5 \mathrm{~min}$, the cells were analyzed using a FluoView FV1000 confocal laser scanning microscope (Olympus Corporation, Tokyo, Japan). The percentage of positively stained cells was calculated in three random fields.

Western blot analysis. Subsequent to collection of the cells, the proteins were extracted using lysis buffer (Beyotime, Shanghai, China), the cell lysates were resolved using SDS-PAGE (Bio-Rad Laboratories, Inc., Hercules, CA, USA) and were electrophoretically transferred to nitrocellulose membranes (Bio-Rad Laboratories, Inc.). Subsequent to blocking with $5 \%$ non-fat milk for $1 \mathrm{~h}$ at room temperature, the membranes were incubated with primary antibodies, including Monoclonal mouse anti-rat immunoglobulin $\mathrm{G}(\operatorname{IgG}) \beta$-actin $(1: 10,000$; Sigma-Aldrich) and anti-LC3B $(1: 1,000)$ followed by the horseradish peroxidase-conjugated secondary antibodies (mouse anti-mouse or mouse anti-rabbit IgG; 1:2000; Beyotime, Shanghai, China). Proteins were visualized using a SuperSignal West Dura Chemiluminescent Substrate (Pierce Biotechnology, Inc., Rockford, IL, USA).

Apoptosis assay. The culture medium was changed to serum-free $\alpha$-MEM for $12 \mathrm{~h}$ in order to induce apoptosis. Apoptotic cells were identified by terminal deoxynucleotidyl transferase dUTP nick end labeling (TUNEL) staining using an In Situ Cell Death Detection Kit, Fluorescein (Roche Diagnostics, Indianapolis, IN, USA) according to the manufacturer's instructions. The cells were incubated with DAPI $(0.5 \mu \mathrm{g} / \mathrm{ml})$ for $5 \mathrm{~min}$ and analyzed under a confocal microscope (FluoView FV1000; Olympus Corp.). Green indicated TUNEL-positive cells, and the percentage of positive cells was calculated in three random fields

Statistical analysis. Analysis was performed using SPSS software Version 15.0 (SPSS Inc., Chicago, IL, USA). Quantitative data are presented as the mean \pm standard deviation and compared using Student's t-test to determine the significance between two groups or one-way analysis of variance followed by a post hoc test among three or more groups. $\mathrm{P}<0.05$ was considered to indicate a statistically significant difference.

\section{Results}

Autophagy was induced in BMSCs from GIOP rats. Prior to testing for autophagy, the proliferative ability of BMSCs in 
A

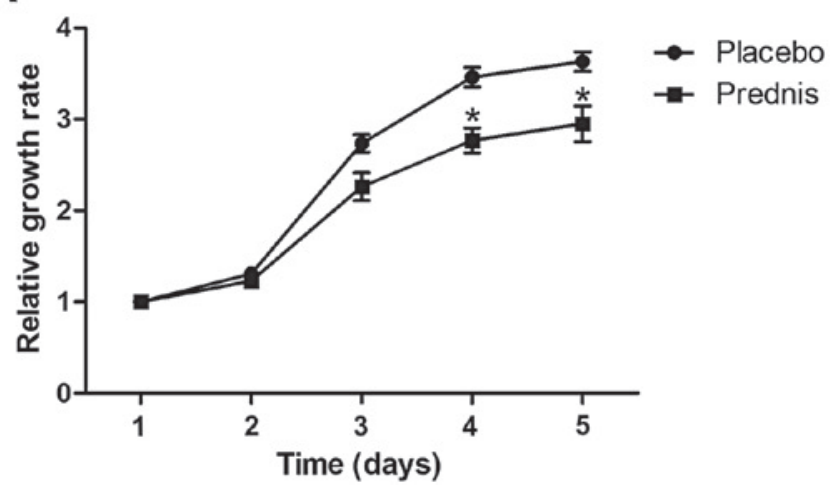

B

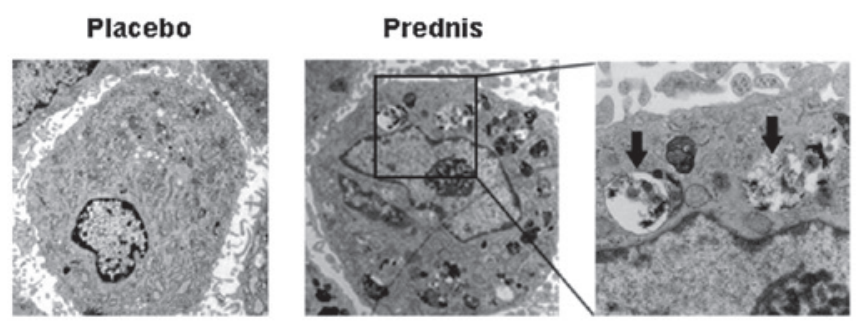

Figure 1. BMSCs of GIOP rats exhibited a reduced proliferative ability and increased autophagy. (A) The relative growth rate curve of BMSCs treated with placebo or prednis for 8 weeks. ${ }^{*} \mathrm{P}<0.05$ vs. placebo. All data are presented as the mean \pm standard deviation and represents the results of three separate experiments. (B) Fixed cells processed for thin-section electron microscopy, arrows indicate the autophagic vacuoles digesting organelles or cytosolic contents (magnification, x2,500). BMSC, bone marrow mesenchymal stem cell; GIOP, glucocorticoid-induced osteoporosis; prednis, prednisolone.
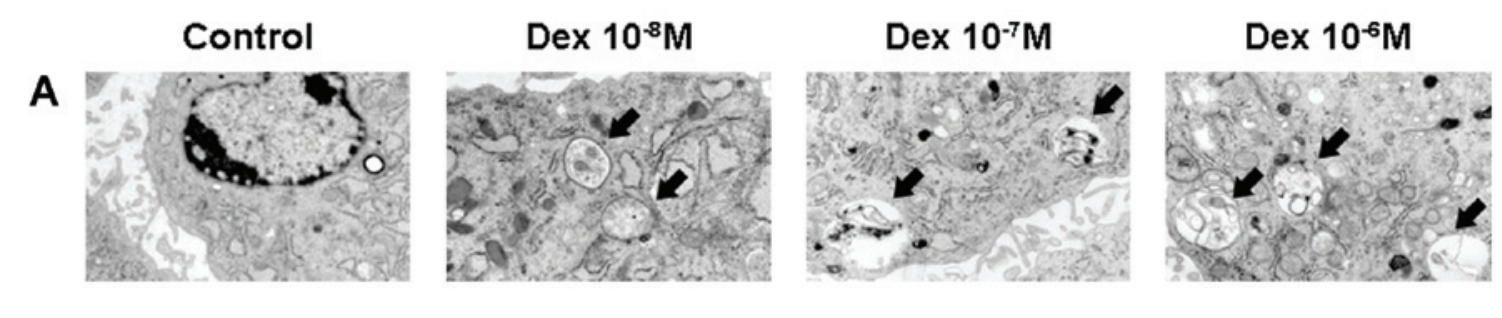

B
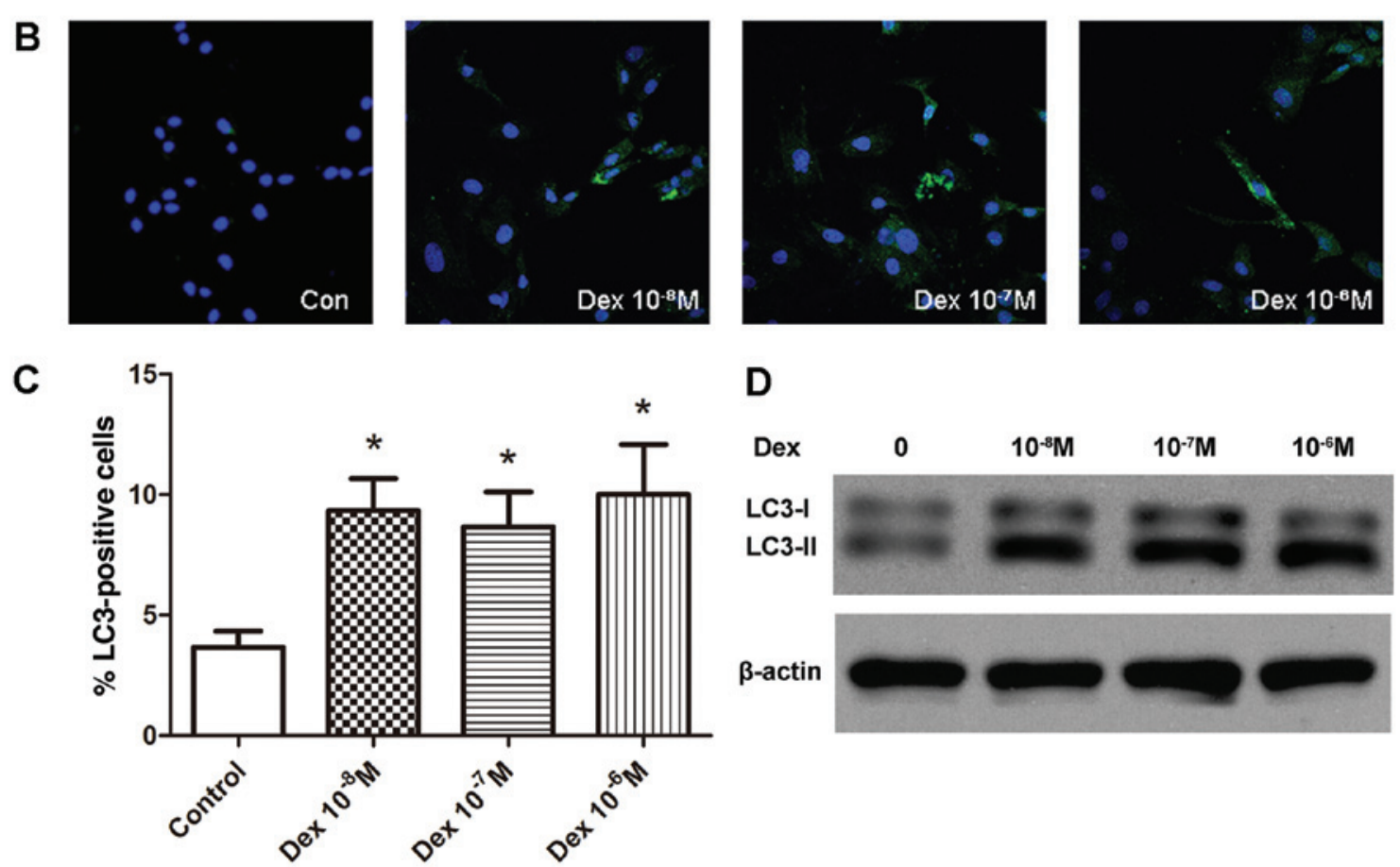

Figure 2. Induction of autophagy in BMSCs in vitro by Dex. (A) Representative micrographs demonstrating an increased number of autophagosomes in all Dex-treated groups compared with the control group. The arrows indicate the autophagosomes (magnification, x6,000). (B) Confocal microscopic analysis of BMSCs following immunofluorescent staining using an anti-LC3 antibody and labeled with the nuclear marker DAPI (magnification, x400). LC3, green; nuclei, blue. (C) Number of LC3-positive cells as a percentage of the positively stained BMSCs. " $\mathrm{P}<0.05$ vs. control, all data are presented as the mean \pm standard deviation and represents the results of three separate experiments.. (D) BMSCs were subject to immunoblotting analysis using anti-LC 3 or anti- $\beta$-actin antibodies, and the autophagic marker LC3-II was induced by various concentrations of Dex. BMSC, bone marrow mesenchymal stem cell; Dex, dexamethasone; LC3, light chain 3.

GIOP rats was compared with that of control rats. CCK-8 was used to determine cell number. The number of BMSCs in the GIOP group was lower than in the placebo group, suggesting a reduced proliferative ability (Fig. 1A). The relative growth rates on days four and five were significantly lower than those in the placebo group $(\mathrm{P}<0.05)$. Using a transmission electron 


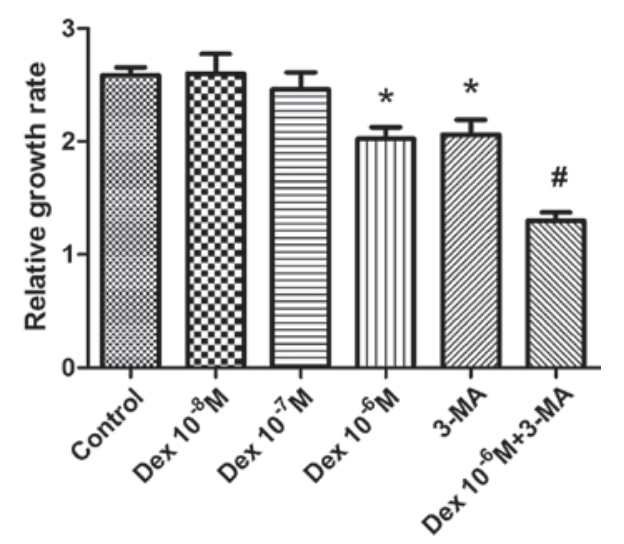

Figure 3. Proliferative ability of BMSCs treated with Dex in the absence or presence of 3-MA. Relative growth rate compared with that prior to treatment with Dex. ${ }^{*} \mathrm{P}<0.05$ vs. control, ${ }^{,} \mathrm{P}<0.01$ vs. $10^{-6} \mathrm{M}$ Dex. All data are presented as the mean \pm standard deviation and represents the results of three separate experiments.. BMSC, bone marrow mesenchymal stem cell; Dex, dexamethasone; 3-MA, 3-methyladenine.

microscope, autophagy was observed in the BMSCs of the GIOP group, but not in those of the placebo group (Fig. 1B).

Dex induced autophagy in BMSCs in vitro. To further elucidate whether glucocorticoids induce autophagy in BMSCs, the BMSCs were treated with three concentrations of Dex $\left(10^{-8} \mathrm{M}, 10^{-7} \mathrm{M}\right.$ and $\left.10^{-6} \mathrm{M}\right)$. Following 48 -h culture with Dex, autophagy was detected by TEM. Autophagy was morphologically characterized by the formation of autophagic vacuoles, also termed autophagosomes. Thin-section electron microscopic analysis identified the accumulation of autophagosomes in all Dex-treated groups, whereas they were scarcely observed in the control group (Fig. 2A). When autophagy occurs, microtubule-associated protein light chain 3 (LC3) is recruited and aggregates in the cytoplasm, thus the expression of LC3 and the increased level of LC3-II are important markers of autophagy. Immunofluorescence analysis demonstrated that treatment with Dex increased the number of LC3-positive cells (Fig. 2B and C). Compared with the control group $(3.7 \pm 1.2 \%)$, the percentage of LC3-positive cells was significantly increased in all Dex-treated cell groups $\left(9.3 \pm 2.3 \%, 8.7 \pm 2.6 \%\right.$ and $10.8 \pm 3.6 \%$ in the $10^{-8}, 10^{-7}$ and $10^{-6} \mathrm{M}$ groups, respectively; $\left.\mathrm{P}<0.05\right)$. The results from the western blot analysis were consistent with the immunofluorescence analysis, demonstrating a greater LC3-II:LC3-I ratio in Dex-treated groups compared with the control (Fig. 2D).

Autophagy maintained the proliferation ability of BMSCs. To determine the effect of glucocorticoid administration on the proliferation of BMSCs, the BMSCs were treated with three concentrations of Dex $\left(10^{-8} \mathrm{M}, 10^{-7} \mathrm{M}\right.$ or $\left.10^{-6} \mathrm{M}\right)$ for $48 \mathrm{~h}$ and then proliferation was evaluated by CCK- 8 assay. The $10^{-6} \mathrm{M}$ concentration of Dex was observed to significantly inhibit the proliferation and number of BMSCs $(\mathrm{P}<0.05)$, while $10^{-8}$ and $10^{-7} \mathrm{M}$ Dex did not produce significant alterations in proliferation, compared with the control group (Fig. 3). The autophagy inhibitor 3-MA was used to investigate the involvement of autophagy in the damage resulting from Dex treatment of BMSCs. The addition of 3-MA was observed to further reduce
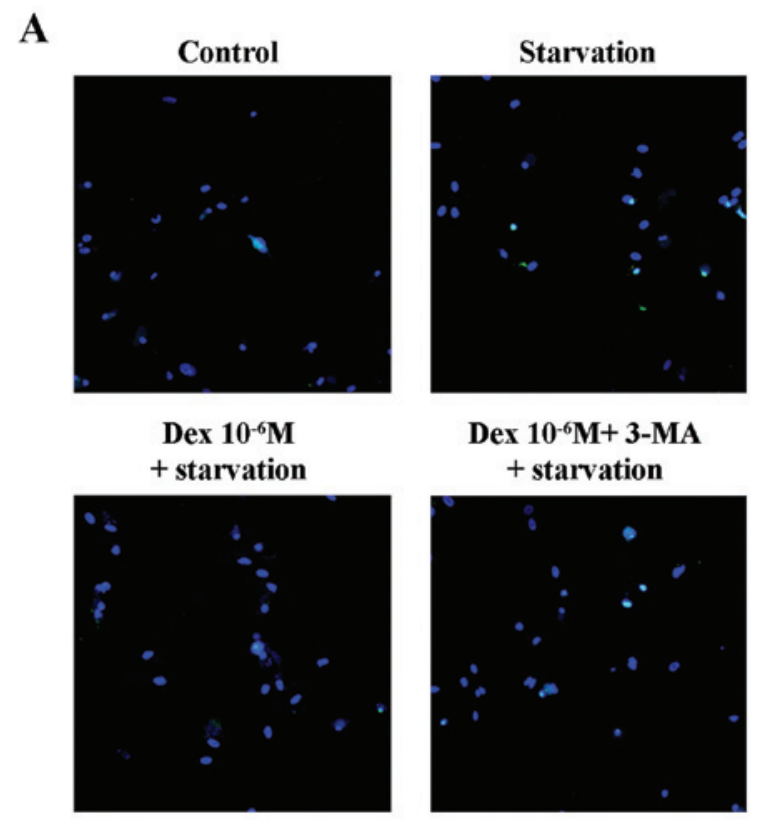

B

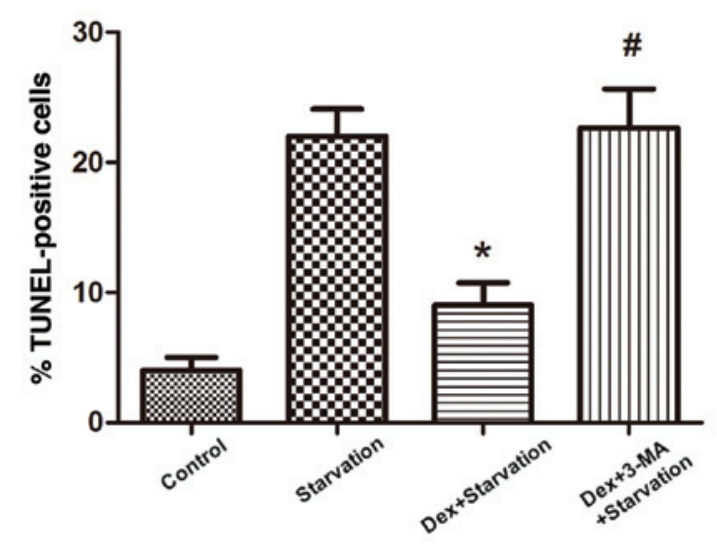

Figure 4. Protective effects of Dex BMSCs from serum deprivation-induced apoptosis. (A) Confocal microscopic analysis of BMSCs following TUNEL staining and labeling with the nuclear marker DAPI (magnification, $x 400$ ). (B) Number of TUNEL-positive cells as a percentage of the positively stained BMSCs. ${ }^{*} \mathrm{P}<0.01$ vs. starvation group, ${ }^{\#} \mathrm{P}<0.05$ vs. Dex + starvation group. All data are presented as the mean \pm standard deviation and represents the results of three separate experiments.. Dex, dexamethasone; BMSC, bone marrow mesenchymal stem cell; TUNEL, terminal deoxynucleotidyl transferase dUTP nick end labeling; 3-MA, 3-methyladenine.

proliferation compared with the control group $(\mathrm{P}<0.05)$, indicating that autophagy partially maintained the proliferation of BMSCs. In addition, the relative growth rate was significantly reduced when BMSCs were co-treated with $10^{-6} \mathrm{M}$ Dex and 3-MA, suggesting that autophagy protected BMSCs from the negative effects on proliferation resulting from Dex treatment.

Autophagy protects against apoptosis in BMSCs. To investigate the effect of autophagy induced by Dex on apoptosis in BMSCs, the culture medium was changed to serum-free $\alpha$-MEM for $12 \mathrm{~h}$ to induce apoptosis subsequent to culture with $10^{-6} \mathrm{M}$ Dex in the absence or presence of 3-MA (Fig. 4). TUNEL assay was used to detect the apoptotic cells. The results demonstrated that, compared with the starvation group $(22.8 \pm 3.6 \%), 10^{-6} \mathrm{M}$ Dex significantly reduced the size of the population of apoptotic BMSCs $(9.3 \pm 2.9 \% ; \mathrm{P}<0.01)$. The use of 
3-MA abrogated the anti-apoptotic effects of Dex, as identified by the increase in apoptotic cells $(22.6 \pm 5.2 \%$; $\mathrm{P}<0.05)$. These results suggest that Dex protected BMSCs from the serum deprivation-induced apoptosis, and that autophagy induced by Dex may be a possible mechanism for this.

\section{Discussion}

Glucocorticoids have been widely used in the treatment of non-infectious inflammation and autoimmune disorders. However, the side effects of glucocorticoid therapy contribute to low bone mass and the occurrence of bone fracture, via disruption to the metabolism and homeostasis of bone tissue. The current study demonstrated that the proliferative ability of BMSCs derived from GIOP rats and Dex-treated BMSCs was impaired. In addition, autophagy was observed in the two types of BMSCs, and the inhibition of autophagy resulted in a further reduction in proliferation. Furthermore, Dex treatment alleviated the levels of apoptosis in starvation-induced BMSCs. These results suggest that autophagy, induced by glucocorticoid therapy, was self-protective in the maintenance of BMSCs.

A previous study using similar methods established that the bone density, the trabecular bone mass and the capacity to form new bone were reduced in GIOP model rats compared with controls (17). In the current study, the proliferative ability of GIOP-BMSCs was observed to be impaired, suggesting that long-term oral glucocorticoid therapy impairs the functional activity of BMSCs. This was hypothesized to be the cause of the reduction in bone formation in the GIOP model. Also, the appearance of autophagy in GIOP-BMSCs indicated that the application of glucocorticoids put BMSCs under stressful conditions.

Dex is a potent glucocorticoid that has been demonstrated to induce autophagy in BMSCs in several experiments (18), the present study examined LC3 in BMSCs using immunostaining and western blot analysis. LC3 was observed to accumulate in the cytoplasm, and the ratio of LC3-II:LC3-I increased with the addition of Dex. LC3-II and LC3-I are important indicators of autophagy.

The results of the current study demonstrated that all three concentrations of Dex $\left(10^{-8} \mathrm{M}, 10^{-7} \mathrm{M}\right.$ and $\left.10^{-6} \mathrm{M}\right)$ were able to significantly increase the occurrence of autophagy, but not in a dose-dependent manner. This observation was in line with the results of a study by Xia et al (15), which identified that Dex was able to induce autophagy in osteocytes.

Dex was used to stimulate the differentiation of BMSCs. However, the regulatory effects of Dex on BMSC proliferation have been reported to be diverse, depending on the different dosage and species used $(19,20)$. The results of the current study demonstrated that $10^{-8}$ and $10^{-7} \mathrm{M}$ doses of Dex did not affect the proliferation of BMSCs, however, $10^{-6} \mathrm{M}$ Dex resulted in a significant reduction. This observation indicates that a high dosage of Dex inhibited the proliferation of BMSCs, which is consistent with the observations in the GIOP-BMSCs.

A study by Oliver et al (21) demonstrated that hBMSCs exhibited a high level of constitutive autophagy and that the knockdown of B-cell lymphoma-XL suppressed autophagy, influencing the survival and differentiation of the hBMSCs. To confirm the role of autophagy in the maintenance of BMSC proliferation, 3-MA (an inhibitor of autophagy) was used. The application of 3-MA was observed to result in a reduction in BMSC survival, suggesting a protective effect of autophagy. In the current study, autophagy alleviated the negative effect of Dex on BMSC proliferation.

Dex has been previously demonstrated to exhibit an effect on the apoptosis of cells $(22,23)$, and it has also been identified to inhibit confluence-induced apoptosis of hBMSCs and negatively regulate the expression of apoptosis-associated genes $(24,25)$. The crosstalk between autophagy and apoptosis is complex and crucial in the determination of cell fate (26). A study by Zhang et al (27) reported that autophagy was able to protect MSCs from apoptosis induced by low oxygen, in addition to serum deprivation. The results of the current study demonstrated that Dex treatment protected against starvation-induced apoptosis to a certain degree. However, Wang et al (28) observed an exacerbation of starvation-induced apoptosis with the administration of $10^{-6} \mathrm{M}$ Dex. This discrepancy may be due to the different time-courses of Dex treatment and cell types used in the different studies.

Autophagy, as mentioned, has been reported to maintain cell activity through removing damaged cellular components. However, a study reported that Dex-induced autophagy leads to cell death due to the destruction of certain components necessary for cell survival (29). The end result of autophagy may depend on the type of cells involved and the severity or time-course of stimuli (30). The results of the current study suggested that upon stimulation with $10^{-6}$ M Dex, autophagy protected BMSCs from apoptosis. However, whether autophagy remained protective or became detrimental when the glucocorticoid dose was increased, or the time-course was prolonged, remains unclear. Whether or not autophagy-induced cell death is involved in the pathogenesis of osteoporosis requires further investigation.

In summary, for the first time, to the best of our knowledge, glucocorticoid therapy was demonstrated to induce autophagy in BMSCs and maintain the proliferative ability of BMSCs. Autophagy induced by Dex may protect BMSCs from starvation-induced apoptosis, suggesting this as a survival mechanism against cell-death. Thus, the regulation of autophagy should be considered as a novel strategy to enhance the activity of BMSCs and increase bone mass in GIOP.

\section{Acknowledgements}

The current study was supported by the Ministry of Science and Technology of China (grant no. 2011CB964703); National High Technology Research and Development Program 863 (grant no. 2012AA020502); and the China Postdoctoral Science Foundation (grant nos. 20100480093 and 2012T50856).

\section{References}

1. Saag KG, Shane E, Boonen S, et al: Teriparatide or alendronate in glucocorticoid-induced osteoporosis. N Engl J Med 357: 2028-2039, 2007

2. Weinstein RS: Glucocorticoid-induced osteoporosis and osteonecrosis. Endocrinol Metab Clin North Am 41: 595-611, 2012.

3. Fraser LA and Adachi JD: Glucocorticoid-induced osteoporosis: treatment update and review. Ther Adv Musculoskelet Dis 1: $71-85,2009$. 
4. O'Brien CA, Jia D, Plotkin LI, et al: Glucocorticoids act directly on osteoblasts and osteocytes to induce their apoptosis and reduce bone formation and strength. Endocrinology 145: $1835-1841,2004$

5. Jia D, O'Brien CA, Stewart SA, etal: Glucocorticoids act directly on osteoclasts to increase their life span and reduce bone density. Endocrinology 147: 5592-5599, 2006.

6. Kanis JA, Johansson H, Oden A, et al: A meta-analysis of prior corticosteroid use and fracture risk. J Bone Miner Res 19: 893-899, 2004

7. Long MW: Osteogenesis and bone-marrow-derived cells. Blood Cells Mol Dis 27: 677-690, 2001.

8. Miura M, Chen XD, Allen MR, et al: A crucial role of caspase-3 in osteogenic differentiation of bone marrow stromal stem cells. J Clin Invest 114: 1704-1713, 2004.

9. Zhou Z, Apte SS, Soininen R, et al: Impaired endochondral ossification and angiogenesis in mice deficient in membrane-type matrix metalloproteinase I. Proc Natl Acad Sci USA 97: 4052-4057, 2000.

10. Wong AS, Cheung ZH and Ip NY: Molecular machinery of macroautophagy and its deregulation in diseases. Biochim Biophys Acta 1812: 1490-1497, 2011.

11. Burman C and Ktistakis NT: Autophagosome formation in mammalian cells. Semin Immunopathol 32: 397-413, 2010.

12. Hocking LJ, Mellis DJ, McCabe PS, Helfrich MH and Rogers MJ: Functional interaction between sequestosome-1/p62 and autophagy-linked FYVE-containing protein WDFY3 in human osteoclasts. Biochem Biophys Res Commun 402: 543-548, 2010.

13. Darcy A, Meltzer M, Miller J, et al: A novel library screen identifies immunosuppressors that promote osteoblast differentiation. Bone 50: 1294-1303, 2012

14. Onal M, Piemontese M, Xiong J, et al: Suppression of autophagy in osteocytes mimics skeletal aging. J Biol Chem 288: 17432-17440, 2013.

15. Xia X, Kar R, Gluhak-Heinrich J, et al: Glucocorticoid-induced autophagy in osteocytes. J Bone Miner Res 25: 2479-2488, 2010

16. Vessoni AT, Muotri AR and Okamoto OK: Autophagy in stem cell maintenance and differentiation. Stem Cells Dev 21: 513-520, 2012

17. Folwarczna J, Pytlik M, Sliwiński L, et al: Effects of propranolol on the development of glucocorticoid-induced osteoporosis in male rats. Pharmacol Rep 63: 1040-1049, 2011.

18. Klionsky DJ, Abdalla FC, Abeliovich H, et al: Guidelines for the use and interpretation of assays for monitoring autophagy. Autophagy 8: 445-544, 2012.
19. Both SK, van der Muijsenberg AJ, van Blitterswijk CA, de Boer J and de Bruijn JD: A rapid and efficient method for expansion of human mesenchymal stem cells. Tissue Eng 13: 3-9, 2007.

20. Hong L, Sultana H, Paulius K and Zhang G: Steroid regulation of proliferation and osteogenic differentiation of bone marrow stromal cells: a gender difference. J Steroid Biochem Mol Biol 114: 180-185, 2009

21. Oliver L, Hue E, Priault M and Vallette FM: Basal autophagy decreased during the differentiation of human adult mesenchymal stem cells. Stem Cells Dev 21: 2779-2788, 2012.

22. Oh HY, Namkoong S, Lee SJ, et al: Dexamethasone protects primary cultured hepatocytes from death receptor-mediated apoptosis by upregulation of cFLIP. Cell Death Differ 13: 512-523, 2006

23. Dorscheid DR, Low E, Conforti A, Shifrin S, Sperling AI and White SR: Corticosteroid-induced apoptosis in mouse airway epithelium: effect in normal airways and after allergen-induced airway inflammation. J Allergy Clin Immunol 111: 360-366, 2003.

24. Song IH, Caplan AI and Dennis JE: Dexamethasone inhibition of confluence-induced apoptosis in human mesenchymal stem cells. J Orthop Res 27: 216-221, 2009.

25. Xiao Y, Peperzak V, van Rijn L, Borst J and de Bruijn JD: Dexamethasone treatment during the expansion phase maintains stemness of bone marrow mesenchymal stem cells. J Tissue Eng Regen Med 4: 374-386, 2010

26. Eisenberg-Lerner A, Bialik S, Simon HU and Kimchi A: Life and death partners: apoptosis, autophagy and the cross-talk between them. Cell Death Differ 16: 966-975, 2009.

27. Zhang Q, Yang YJ, Wang H, et al: Autophagy activation: a novel mechanism of atorvastatin to protect mesenchymal stem cells from hypoxia and serum deprivation via AMP-activated protein kinase/mammalian target of rapamycin pathway. Stem Cells Dev 21: 1321-1332, 2012.

28. Wang H, Pang B, Li Y, Zhu D, Pang T and Liu Y: Dexamethasone has variable effects on mesenchymal stromal cells. Cytotherapy 14: 423-430, 2012.

29. Laane E, Tamm KP, Buentke E, et al: Cell death induced by dexamethasone in lymphoid leukemia is mediated through initiation of autophagy. Cell Death Differ 16: 1018-1029, 2009.

30. Codogno P and Meijer AJ: Autophagy and signaling: their role in cell survival and cell death. Cell Death Differ 12 (Suppl 2): $1509-1518,2005$. 